\title{
Clinical Outcomes of Patients with Pulmonary Agenesis
}

\author{
Shouji Fukuoka ${ }^{1}$, Hazumu Nagata ${ }^{1}$, Kenichiro Yamamura ${ }^{1}$, Daisuke Toyomura ${ }^{1}$, Yusaku \\ Nagatomo $^{1}$, Yoshimi Eguchi ${ }^{1}$, Kiyoshi Uike ${ }^{1}$, Yuichiro Hirata ${ }^{1}$, Hirosuke Inoue ${ }^{1}$, Masayuki \\ Ochiai $^{1}$, and Shouichi Ohga ${ }^{1}$
}

${ }^{1}$ Kyushu Daigaku

August 25, 2021

\begin{abstract}
Introduction: Pulmonary agenesis is a complete absence of the pulmonary parenchyma, bronchi beyond the bifurcation, and pulmonary vasculature unilaterally or bilaterally. Because of the rare occurrence, its pathophysiology and outcome remain elusive. We evaluate the clinical features and risk factors for mortality due to pulmonary agenesis. Methods: Two patients we experienced are presented as index cases. All reported cases of pulmonary agenesis were collected from online and publication databases between 1955 and 2020. We assessed the impact of comorbidity and intervention on the survival outcome. Results: We identified 230 patients - 138 (60\%) with right-sided, and $14(6 \%)$ with bilateral agenesis - among 164 articles and our cases. There were $93(40 \%)$ cardiovascular, 70 (30\%) skeletal, and 48 (21\%) gastrointestinal anomalies; 47 (20\%) tracheal stenoses; and $33(14 \%)$ genitourinary anomalies. Fifty-two (23\%) patients had isolated pulmonary agenesis. The 2-year overall survival (OS) rate was $66 \%$ and there was no subsequent death until 13 years of age. The right agenesis was significantly associated with a lower 2 -year OS rate $(58 \%$ vs. $78 \%, \mathrm{p}=0.019)$ or more frequent tracheal stenosis $(28 \%$ vs. $12 \%, \mathrm{p}=0.006)$ than left-sided disease. A multivariable analysis indicated that tracheal stenosis (hazard ratio [HR] 2.4, 95\% confidence interval [CI] 1.5-4.1, $\mathrm{p}=0.004$ ) and gastrointestinal anomaly (HR 1.9, 95\% CI 1.1-3.3, $\mathrm{p}=0.018$ ) were prognostic factors for mortality. Conclusions: The poor prognostic factors were tracheal stenosis, right agenesis and gastrointestinal anomaly. Tracheal and gastrointestinal controls are targeted at birth and in infancy for the improved survival of unilateral pulmonary agenesis.
\end{abstract}

\section{Hosted file}

0801 Final Draft.docx available at https://authorea.com/users/431767/articles/535253clinical-outcomes-of-patients-with-pulmonary-agenesis

\section{Hosted file}

PulmoAgen Table.docx available at https://authorea.com/users/431767/articles/535253-clinicaloutcomes-of-patients-with-pulmonary-agenesis 

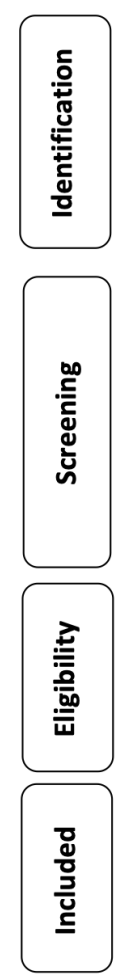
Total 466 articles identified from electronic searches to capture primary
articles on pulmonary agenesis

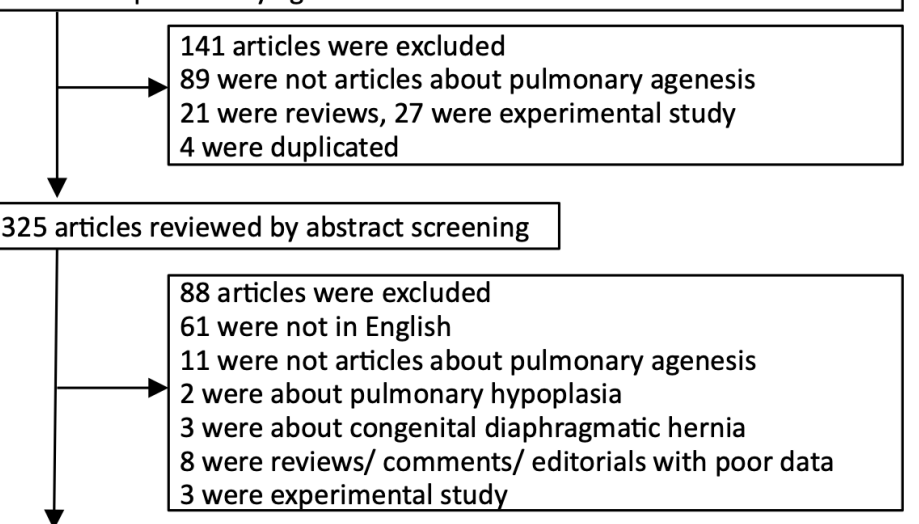

237 articles reviewed by full text screening

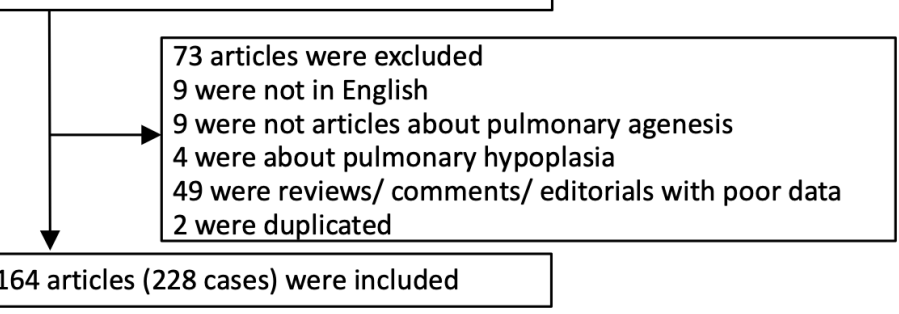

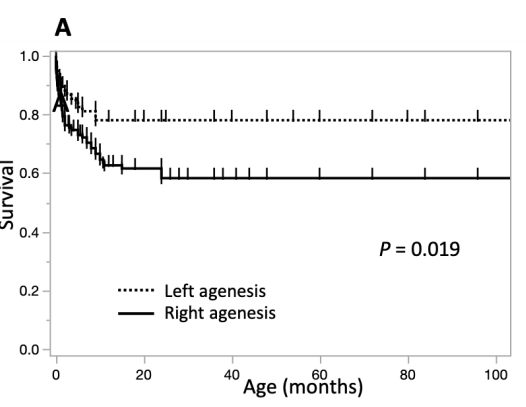

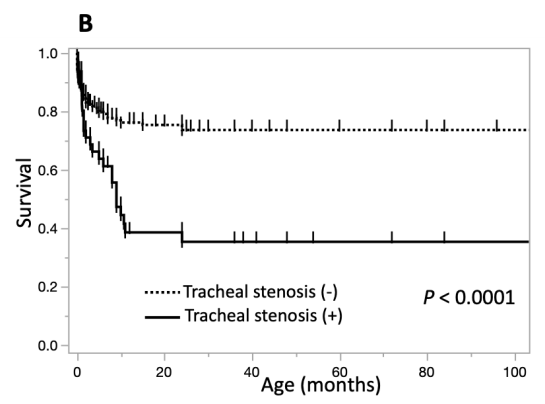



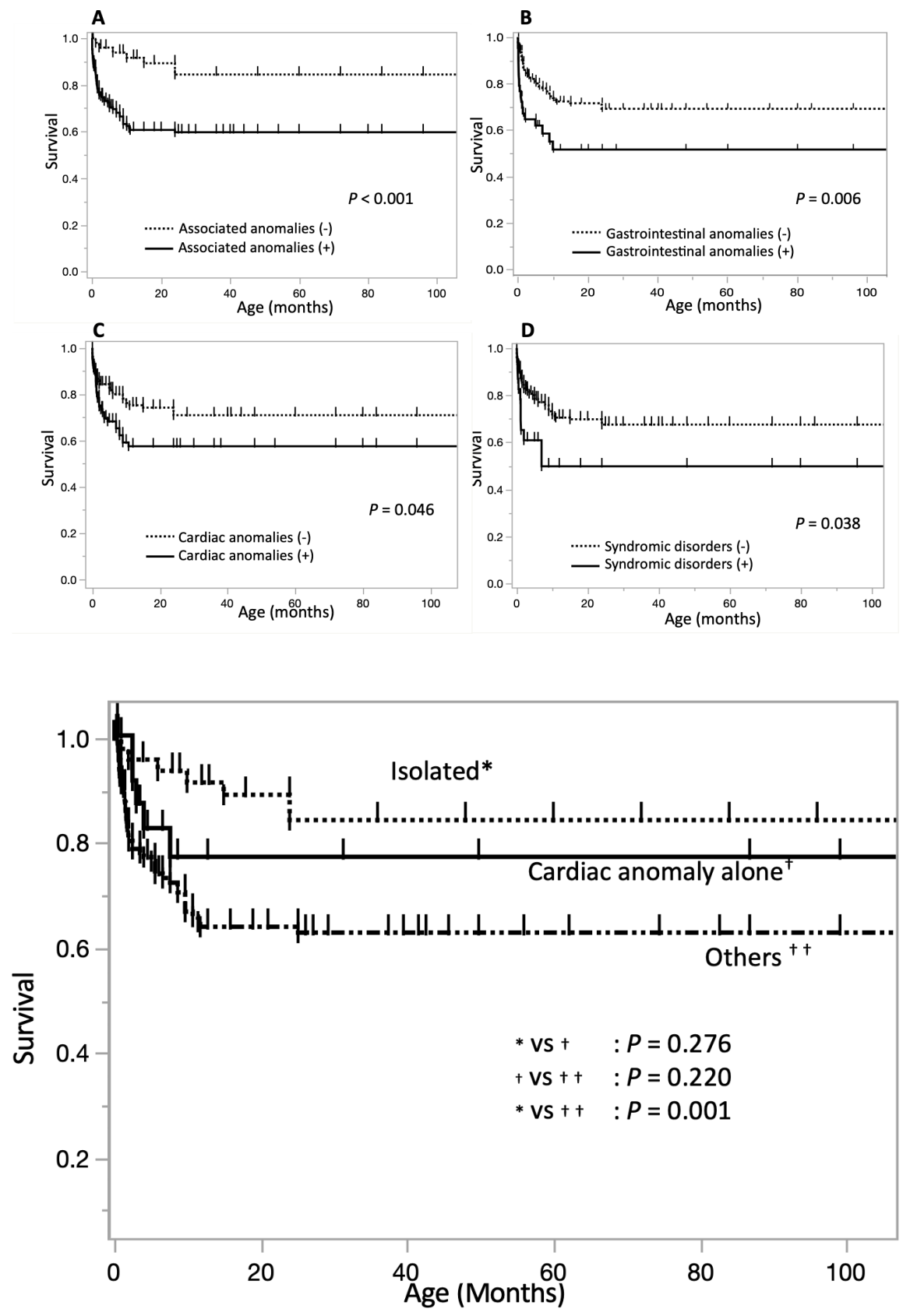